\title{
The Electronic Structure of the Cobalt(III) Hexammine Complex Ion: A Semi-empirical Molecular Orbital Calculation
}

\author{
THOMAS H. WIRTH*
}

Department of Physical Chemistry, The University of Copenhagen, Copenhagen, Denmark

\begin{abstract}
Using the extended Wolfsberg-Helmholz method proposed by Ballhausen and Gray a semi-empirical molecular orbital calculation has been performed on the $\mathrm{Co}\left(\mathrm{NH}_{3}\right)_{6}{ }^{3+}$ ion. The derived energy level scheme cannot directly be compared with the experimental value of $10 D q$, but the calculated splitting of the $d$-orbitals has the right order of magnitude.
\end{abstract}

$\mathrm{T}_{\mathrm{s}}^{\mathrm{b}}$ he LCAO-MO theory is an attractive conceptual basis on which to discuss bonding in transition metal complexes. The quantitative application of the molecular orbital method to even the simplest complexes, however, presents very difficult computational problems if one begins from first principles. ${ }^{1}$ The semi-empirical approach introduced by Wolfsberg and Helmholz, ${ }^{2}$ on the other hand, permits radical simplifications. These simplifications have made it possible to apply the LCAO-MO formalism to a variety of complex ions, including the vanadyl ion, ${ }^{3}$ the permanganate ion,,$^{4,5}$ the hexafluorotitanate(III) ion, ${ }^{6}$ and a series of maleonitrile dithiolate complexes. ${ }^{7}$

Perhaps the most elaborate extension of the Wolfsberg-Helmholz semiempirical method is that of Ballhausen and Gray. ${ }^{3}$ The details of this technique have been presented in their recent book. ${ }^{8}$

The semi-empirical method has not been universally successful. Cotton and Haas have computed the $d$-orbital splittings in a series of $\mathrm{M}\left(\mathrm{NH}_{3}\right)_{6}{ }^{n+}$ complexes, ${ }^{11}$ and their results seemed to indicate that quantitative agreement with experiment requires a "judicious adjustment" of assumptions and parameters to fit each case. On more general grounds the Wolfsberg-Helmholz method has also been criticized by Fenske. ${ }^{10}$

It is possible, however, to improve the calculations of Cotton and Haas considerably even while remaining within the framework of the WolfsbergHelmholz approximations. Valence State Ionization Potentials dependent on

* Present address: South Carolina State College, Dept. of Nat. Sci., Orangeburg, S.C., U.S.A.

Acta Chem. Scand. 19 (1965) No. 10 
configuration as well as charge and smoothed across the periodic table can be employed..$^{9}$ Ligand-ligand overlap can be included in the calculation.

Better radial wave functions can be used. Approximate cobalt $4 p$ radial wave functions are now available; ${ }^{13}$ Cotton and Haas used the cobalt $4 s$ function in place of the $4 p$ function. A full Hartree-Fock calculation of the ammonia molecule has recently been published by Moccia ${ }^{14}$ using a basis set centered on the nitrogen atom. The resulting wave function of the highest occupied orbital can be used as the ligand sigma orbital; it is surely a great improvement over the simple $s p^{3}$ hybrid used by Cotton and Haas.

The value of the ionization potential of ammonia was taken by Cotton and Haas from an electron impact determination. A lower and more accurate value is available from photoionization data. ${ }^{12}$

The present work is a calculation utilizing these improvements and applied to the $\mathrm{Co}\left(\mathrm{NH}_{3}\right)_{6}{ }^{3+}$ ion.

\section{DETAILS OF THE CALCULATION}

The cobalt(III) hexammine ion is a regular octahedron of $O_{h}$ symmetry. The construction of symmetry orbitals and factorization of the secular determinant for such complexes has been described many times (see, e.g., Ref. 15).

The radial functions used to evaluate the group overlap integrals were obtained from Hartree-Fock treatments of the cobalt atom and the ammonia molecule. In the ammonia calculation of Moccia, ${ }^{14}$ a basis set of Slater-type functions all centered on the nitrogen atom was used. The resulting $A_{1}$ orbital of highest energy was used as the ligand sigma orbital in the present calculation. In addition to the $s$-, $p$-, $d$-, and $f$-sigma components, this orbital contains a small component proportional to $\sin 3 \varphi$, where $\varphi$ is the angle about the three-fold axis of ammonia. The overlap integrals contain terms involving this component, but those terms vanish if the integrals are averaged over all orientations of the hydrogen atoms.

The cobalt $3 d$ and $4 s$ radial functions used were those of Clementi. ${ }^{17} \mathrm{Un}$ fortunately, full Hartree-Fock calculations are not available for excited configurations of the transition metals. Therefore the approximate cobalt $4 p$ function of Richardson, Powell, and Nieuwpoort ${ }^{13}$ was used.

The Co- $\mathrm{N}$ distance was taken as $1.90 \AA .{ }^{18}$

The diagonal matrix elements of the Hamiltonian with the cobalt $3 d$, $4 s$, and $4 p$ orbitals were computed as functions of charge and electronic configuration from the Valence State Ionization Energies of Ballhausen and Gray. ${ }^{9}$ As described in Ref. 8, an electronic configuration is assumed, the secular equation solved, and a Mullikan population analysis performed. The process is repeated until the initial and final electron configurations on the metal are identical.

Evaluation of the diagonal matrix element of the Hamiltonian with the ammonia sigma orbital is not straightforward. From the "isolated atom" point of view, this quantity should be the VSIE of the appropriate hybrid orbital of atomic nitrogen, which is a function of charge on the nitrogen atom. The hydrogen atoms, however, compensate in part for the donation of nitrogen electrons to the metal ion. The net result is delocalization of positive charge 
from metal to hydrogen. The charge on nitrogen cannot be computed without knowing the charge on hydrogen. The $\mathrm{N}-\mathrm{H}$ bonds, however, are not included in the population analysis.

In the present calculation, the ligand VSIE was set equal to the ionization potential of ammonia, $10.15 \mathrm{eV}{ }^{12}$ This value is appropriate to the case in which the charge is completely delocalized to the eighteen hydrogen atoms on the surface of the complex. The charge on nitrogen is then the same in the complex as in free ammonia.

To test the effect of modifying the ammonia VSIE, a number of selfconsistent calculations were performed with different values of this parameter. The metal VSIE and the one-electron energies of the molecular orbitals were found to be strong linear functions of the assumed ligand VSIE. The energy differences between one-electron energies also vary linearly with the ammonia VSIE. The calculated value of the splitting of the $d$-orbitals, however, is not nearly so sensitive to changes in the ammonia VSIE as are the single oneelectron energies.

Ligand-ligand overlap is significant. The overlap between sigma orbitals centered on adjacent ligands is 0.0975 ; between orbitals centered on opposite sides of the metal atom the overlap is $\mathbf{0 . 0 3 0 3}$. The group overlap integrals are presented in Table 1. Ligand-ligand overlap is neglected in Case I; in

Table 1. Group overlap integrals for $\mathrm{Co}$ (III) hexammine.

\begin{tabular}{llll}
\hline & \multicolumn{3}{c}{ Group overlap integrals } \\
Calculation & $e_{g}$ & $a_{g}$ & $t_{1 u}$ \\
\hline Case I & 0.172 & 0.680 & 0.231 \\
Case II & 0.189 & 0.571 & 0.235 \\
Cotton and Haas (11) & 0.180 & 0.754 & 0.440 \\
Yamatera (19) & 0.3 & 0.72 & 0.6
\end{tabular}

Case II it is included. For comparison, the values used in the calculations of Yamatera ${ }^{19}$ and Cotton and Haas ${ }^{11}$ are presented as well.

The present integrals differ from those of the previous authors chiefly in the value of the $t_{1 u}$ group overlap integral. This difference is not surprising. Cotton and Haas used a $4 s$ radial function rather than a $4 p$ function in computing the integral. Yamatera used simple Slater atomic orbitals as basis functions.

The roots of the secular determinant were obtained for two separate cases. In one case ligand-ligand overlap was neglected; in the other, ligand-ligand overlap corrections were included. In each case the repeated calculations necessary to obtain a self-consistent distribution of electrons were performed by computer, using a program prepared by $\mathbf{H}$. Johansen. ${ }^{20}$ The WolfsbergHelmholz factor $F$, where $H_{i j}=-F S_{i j} \sqrt{H_{i i} H_{j j}}$, was set equal to 2.00 . (In this formula $H_{i j}$ is $\left\langle\psi_{i}|H| \psi_{j}\right\rangle$ and $S_{i j}$ is $\left\langle\psi_{i} \mid \psi_{j}\right\rangle$ ). The computed energy levels are displayed in Figs. 1 and 2. The one-electron orbitals and their energies which result if ligand-ligand overlap is included are arranged in order of decreasing energy in Table 2. 


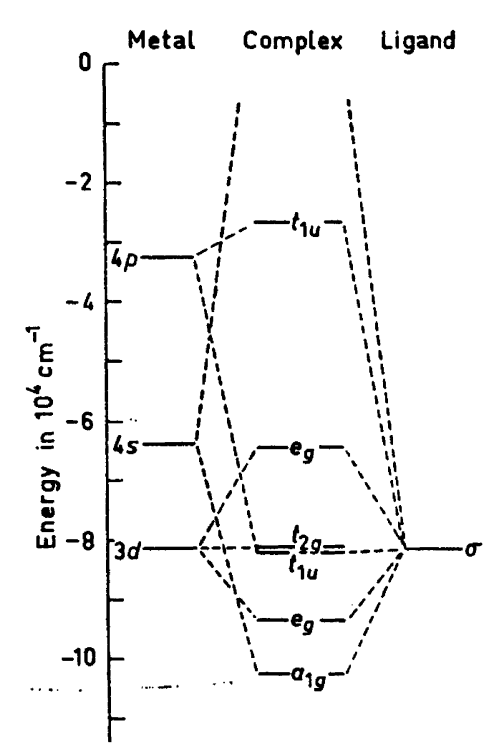

Fig. 1. $\mathrm{Co}\left(\mathrm{NH}_{3}\right)_{6}^{3+}$. One-electron energies. Ligand-ligand overlap neglected.

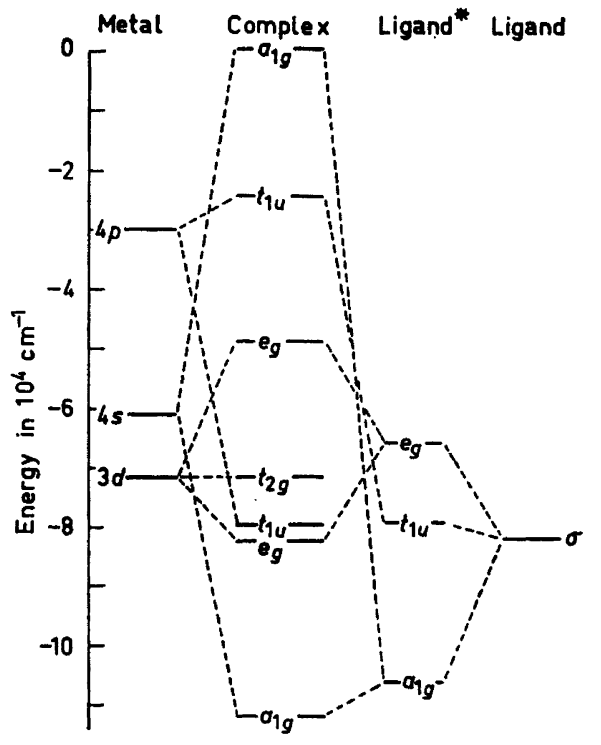

Fig. 2. $\mathrm{Co}\left(\mathrm{NH}_{3}\right)_{6}{ }^{3+}$. One-electron energies. Ligand-ligand corrections included.

* Corrected for ligand-ligand overlap.

If ligand-ligand overlap is neglected, the computed energy separation between the $t_{2 g}$ and the $e_{g}$ levels is $1.68 \times 10^{4} \mathrm{~cm}^{-1}$. If ligand-ligand overlap is included in the calculation, the result is $2.28 \times 10^{4} \mathrm{~cm}^{-1}$.

Table 2. Molecular orbitals of $\mathrm{Co}\left(\mathrm{NH}_{3}\right)_{8}^{3+}$. (Including ligand-ligand overlap).

\begin{tabular}{ccc}
\hline Orbital & Energy $\left(10^{4} \mathrm{~cm}^{-1}\right)$ & $\begin{array}{c}\text { Expansion } \\
\text { coefficient } \lambda^{* *}\end{array}$ \\
\hline$a_{1 g}$ & +0.045 & -0.759 \\
$t_{1 u}$ & -2.43 & -0.318 \\
$e_{g}$ & -4.88 & -1.159 \\
$t_{2 g}$ & -7.16 & 0.000 \\
$t_{1 u}$ & -8.98 & 0.805 \\
$e_{g}$ & -11.17 & 3.020 \\
$a_{1 g}$ & & \\
Electron configuration: $3 d^{8.36}$ & $4 s^{0.40}$ & $4 p^{0.17}$ \\
Charge on cobalt: +0.07 & &
\end{tabular}

** $\Psi=N[\psi($ metal $)+\lambda \psi$ (ligand) $]$, where $\Psi$ is a molecular wave function, $N$ is a normalization constant, and the $\psi$ 's are linear combinations of basis orbitals which transform according to $O_{h}$ symmetry. 


\section{DISCUSSION}

The quantity usually referred to as $10 D q$ is by definition given as the energy separation between the single electron orbital energies of $e_{8}^{*}$ and $t_{2 g}$ with no inclusion of $1 / r_{12}$ terms. However, this is not the energy difference calculated above. The reason is that the calculated separation between $e_{g}$ and $t_{2 g}$ is dependent upon the VSIE's, which in turn are values averaged over the different configurations of the gaseous metal ion. Hence the calculated energy difference between $e_{g}$ and $t_{2 g}$ is dependent upon $1 / r_{12}$ terms. How large a contribution these terms give is difficult to assess. If we ignore this effect we get $D q$ equal to $2280 \mathrm{~cm}^{-1}$. This is in good agreement with an estimated crystal field value ${ }^{21}$ of $2460 \mathrm{~cm}^{-1}$. Since, however, the calculated and the "experimental" value for the energy separation of $e_{g}$ and $t_{2 g}$ are estimated using different averaging processes over the various multiplets a closer comparison is not possible.

The fact that the numerical results nevertheless are satisfactory in this simple system does not of course place the semi-empirical method above reproach. ${ }^{10}$ Yet it is encouraging that the results of Cotton and Haas can be improved so significantly by using more accurate wave functions and other input parameters and by modifying their method in logically justifiable ways.

These and other calculations have shown that it is necessary to exercise great caution in applying the approach to complicated ions of low symmetry.22 Discussion of bonding and assignment of transitions in such ions often requires that electron repulsion and configuration interaction be taken into account. It is sometimes necessary to calculate transition moments, which may be small even though the transition is allowed by the overall symmetry of the molecule. It is doubtful that the central approximations of the extended Wolfsberg-Helmholz approach are accurate enough to support these elaborate superstructures.

Acknowledgements. The author was supported at the University of Copenhagen by a Fulbright-Hays Grant. He is deeply indebted to Mr. Helge Johansen, who performed much of the computer work. He would like to thank Professor C. J. Ballhausen for several suggestions, Dr. Gerd LaMar for many helpful discussions, and Dr. Philip Stephens for a correction.

\section{REFERENCES}

1. Shulman, R. G. and Sugano, S. Phys. Rev. Letters 7 (1961) 157.

2. Wolfsberg, M. and Helmholz, L. J. Chem, Phys. 20 (1952) 837.

3. Ballhausen, C. J. and Gray, H. B. Inorg. Chem. 1 (1962) 111.

4. Fenske, R. F. and Sweeney, C. C. Inorg. Chem. 3 (1964) 1105.

5. Viste, A. and Gray, H. B. Inorg. Chem. 3 (1964) 1113.

6. Bedon, H. D., Horner, S. M. and Tyree, S. Y., Jr. Inorg. Chem. 3 (1964) 647.

7. Shupack, S. I., Billig, E., Clark, R. J. H., Williams, R. and Gray, H. B. J. Am. Chem. Soc. 86 (1964) 4594.

8. Ballhausen, C. J. and Gray, H. B. Molecular Orbital Theory, Benjamin 1964, pp. $92-131$.

9. Ibid. pp. 120-121.

* Corrected for ligand-ligand overlap.

Acta Chem. Scand. 19 (1965) No. 10 
10. Fenske, R. F. Inorg. Chem. 4 (1965) 33.

11. Cotton, F. A. and Haas, T. E. Inorg. Chem. 3 (1964) 1004.

12. Watanabe, K. and Mottl, J. R. J. Chem. Phys. 26 (1957) 1773.

13. Richardson, J. W., Powell, R. R. and Nieuwpoort, W. C. J. Chem. Phys. 38 (1963) 796.

14. Moccia, R. J. Chem. Phys. 40 (1964) 2176.

15. Ballhausen, C. J. Introduction to Ligand Field Theory, McGraw-Hill, New York 1962, pp. $152-163$.

16. Ibid. p. 162.

17. Clementi, E. J. Chem. Phys. 41 (1964) 295.

18. Meisel, K. and Tiedje, W. Z. anorg. allgem. Chem. 164 (1927) 223.

19. Yamatera, H. J. Inst. Polytech. Osaka City Univ. 5 (1956) 163.

20. Johansen, H. Algol Programs for Molecular Calculations, Chemical Laboratory IV, University of Copenhagen 1965, pp. 20-32. Available on request.

21. Ballhausen, C. J. Introduction to Ligand Field Theory, McGraw-Hill, New York 1962 , p. 260, and references therein,

22. Wentworth, R. A. D. and Piper, T. S. Inorg. Chem. 4 (1965) 709.

Received August 9, 1965. 